
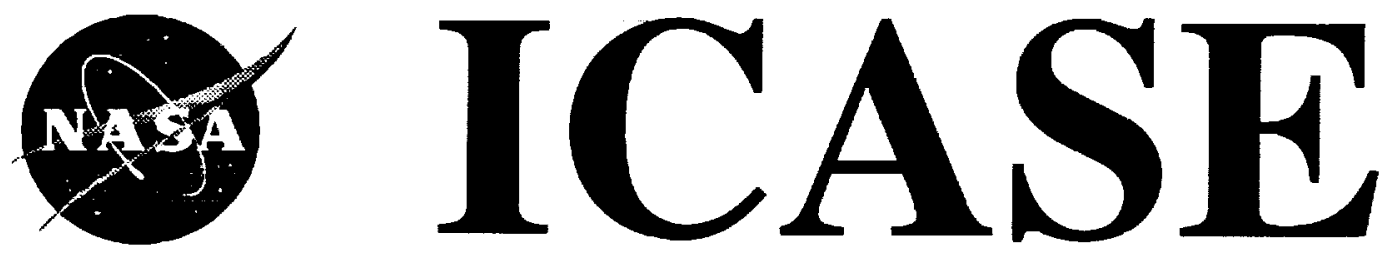

TIME-EVOLVING BUBBLES IN

TWO-DIMENSIONAL STOKES FLOW

Saleh Tanveer

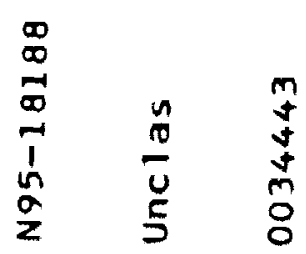

Giovani L. Vasconcelos

Contract NAS1-19480

November 1994

Institute for Computer Applications in Science and Engineering NASA Langley Research Center

Hampton, VA 23681-0001

SRA Operated by Universities Space Research Association

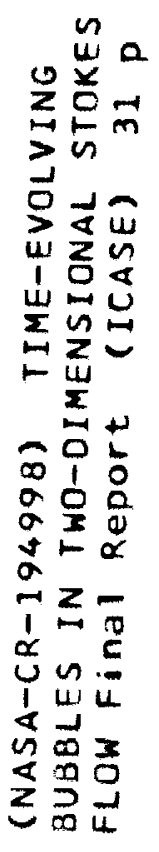




\title{
Time-evolving bubbles in two-dimensional Stokes flow
}

\author{
Saleh Tanveer ${ }^{1}$ and Giovani L. Vasconcelos \\ Department of Mathematics \\ Ohio State University \\ Columbus, $\mathrm{OH} 43210$
}

\begin{abstract}
A general class of exact solutions is presented for a time evolving bubble in a twodimensional slow viscous flow in the presence of surface tension. These solutions can describe a bubble in a linear shear flow as well as an expanding or contracting bubble in an otherwise quiescent flow. In the case of expanding bubbles, the solutions have a simple behavior in the sense that for essentially arbitrary initial shapes the bubble will asymptote an expanding circle. Contracting bubbles, on the other hand, can develop narrow structures ('near-cusps') on the interface and may undergo 'break up' before all the bubble-fluid is completely removed. The mathematical structure underlying the existence of these exact solutions is also investigated.
\end{abstract}

\footnotetext{
${ }^{1}$ Research was supported in part by the National Aeronautics and Space Administration under NASA Contrac No. NAS1-19480 while the author was in residence at the Institute for Computer Applications in Science and Engineering (ICASE), NASA Langley Research Center, Hampton, VA 23681.
} 
I I 


\section{Introduction}

The study of the deformation and breakup of drops and bubbles in a slow viscous flow is of practical significance to many physical processes such as the rheology of emulsions and the mixing in multiphase viscous systems. Following the pioneering work by G. I. Taylor $(1932,1934)$, there has been a great deal of both theoretical and experimental research on the subject. The reviews by Acrivos (1983) and Rallison (1984) summarize the state of affairs in the early eighties. In many of these early studies the term 'drop breakup' usually does not refer to the fragmentation of a drop, but to the non-existence of a steady solution when the applied shear strength exceeds some critical value. In the past decade, however, there have been a number of mainly experimental and computational investigations of the actual dynamics leading to breakup. These have recently been reviewed by Stone (1994).

Analytical solutions for the time evolution of a general three-dimensional drop or bubble in a slow viscous flow do not appear amenable to currently known techniques. The simplified case of two-dimensional bubble flows, on the other hand, is analytically tractable through complex variable methods; and their study might shed some light on important qualitative aspects of axisymmetric 3D flows. In this vein, Richarson (1968) has obtained exact solutions for an inviscid 2D bubble in a linear shear flow, while Buckmaster \& Flaherty (1973) has found approximate solutions for a drop with the same viscosity as the ambient fluid.

There have also been recent investigations of $2 \mathrm{D}$ Stokes flows with time-dependent free boundaries. In a series of papers, Hopper $(1990,1991,1992,1993)$ has found several analytic solutions for the motion of a blob of viscous fluid driven by surface tension. Among these are solutions describing the coalescence of two cylinders (Hopper 1990) and the coalescence of a cylinder with a half-plane (Hopper 1992)--two problems of interest in viscous sintering. Richardson (1992) has recently reviewed the mathematical structure of these solutions. Howison and Richardson (1993) have also obtained exact solutions for the case of a $2 \mathrm{D}$ viscous blob with $n$-th fold symmetry (i.e., invariance under rotation by $2 \pi / n$, for $n>1$ ) in the presence of suction. In the absence of surface tension their solutions develop cusp singularities on the interface (and hence cease to be physically meaningful) before the fluid is completely removed. A nonzero surface tension, however, allows for total removal of fluid.

The main aim of the present paper is to report a general class of exact solutions for a time-evolving bubble in a 2D Stokes flow. Our solutions include, for instance, the motion of a bubble of essentially arbitrary initial shape in a linear flow or an expanding/contracting bubble in an otherwise quiescent flow. In the former case, one is able to follow the transient motion of the bubble as it approaches the steady state. In an earlier paper (Tanveer \& Vasconcelos 1994a), we briefly discussed the case of specific solutions that show a contracting bubble developing topological singularities ('breakup') before the bubble shrinks to zero 
size. Other solutions show that cusp can form in the presence of certain symmetries when surface tension is neglected-in the presence of surface tension, however, cusp formation is inhibited until all the bubble-fluid ('air') is extracted. It was also pointed out that contrary to the expectations from a Hele-Shaw flow analogy recently made in the literature (Howison and Richardson 1993), a circular bubble expanding in a 2D Stokes flow is stable, while a contracting one is not. Here in this paper, we report a more complete set of such solutions for expanding/contracting bubbles, present detailed analysis of the 'regularization' effects provided by surface tension, and discuss the mathematical structure that, among other properties, guarantees the existence of exact solutions for a rather general class of initial conditions.

The paper is organized as follows: in $\S 2$ the problem is mathematically formulated in terms of a conformal mapping from the interior of a unit circle to the flow domain. It should be noted here that the formulation of the problem of a bubble in a 2D Stokes flow parallels that of a 2D viscous drop, as presented by Hopper (1990) and Richardson (1992). In fact, a shorter presentation in $\S 2$ could have been achieved by quoting some of their formulas. However, since the two problems differ in a number of aspects and since there are differences in the derivations, we felt that a complete formulation was warranted here. In $\S 3$ we discuss certain global properties of the conformal mapping that underlie the existence of exact solutions for the problem. Many of the arguments presented in this discussion transcends the specific details of the problem in question and have been found to apply to other $2 \mathrm{D}$ free-boundary problems as well (Tanveer 1993). Accordingly, this section is likely to appeal to researchers interested in finding exact solutions to free-boundary problems that can be conveniently cast in terms of conformal mapping. For those more interested in the concrete results for a $2 \mathrm{D}$ Stokes bubble, however, it can be skipped without any loss of continuity. In $\S 4$ a general class of exact solutions of polynomial type is presented, while the following two sections give details for two specific cases. First, in $\S 5$ the problem of a bubble placed in a linear flow is considered for two particular flow arrangements of relevance to experiments: $\$ 5.1$ describes a bubble in a pure straining flow, whereas $\$ 5.2$ focuses on a simple shear flow. The case of expanding/contracting bubbles in an otherwise quiescent flow is then addressed in $\S 6$. Our conclusions and main results are summarized in $\S 7$.

\section{Mathematical Formulation}

We consider the problem of a bubble placed in a two-dimensional slow viscous flow. The fluid inside the bubble has a negligible viscosity and is at a constant pressure, which is chosen to be zero without loss of generality. The fluid outside the bubble has a viscosity $\mu$ and is incompressible. Under the assumption of no inertial effects, gravitational or other 
body forces, the fluid motion is governed by the Stokes equation and the incompressibility condition:

$$
\begin{aligned}
\mu \nabla^{2} \mathbf{u} & =-\nabla p \\
\nabla \cdot \mathbf{u} & =0
\end{aligned}
$$

where $\mathbf{u}(x, y)$ is the fluid velocity and $p$ the pressure.

On the bubble boundary we must ensure continuity of the shear stress and satisfy the requirement that the jump in the normal stress across the interface equals the product of the surface tension $\sigma$ and the curvature $\kappa$. These two stress boundary conditions can be written as

$$
-p n_{j}+2 \mu e_{j k} n_{k}=\sigma \kappa n_{j},
$$

where the indexes $j$ and $k$ take on values 1 and 2 (the Einstein summation convention is used in the above), and $n_{1}$ and $n_{2}$ are the $x$ and $y$ component of the outward normal unit vector. Here $e_{j k}$ are the components of the rate of strain tensor and are given by

$$
e_{j k}=\frac{1}{2}\left(\frac{\partial u_{j}}{\partial x_{k}}+\frac{\partial u_{k}}{\partial x_{j}}\right) .
$$

In addition, we must also satisfy the usual kinematic condition that the normal velocity $V_{n}$ of a point on the bubble surface is equal to the normal component of fluid velocity at that point, that is,

$$
\mathbf{u} \cdot \mathbf{n}=V_{n} .
$$

To completely specify the problem we need to prescribe appropriate boundary conditions at infinity. We suppose in general that the bubble is placed in a linear flow and its area is changing at a prescribed rate $m$, which in general can be a time dependent function. More specifically, we require that far away from the bubble the fluid velocity behaves as

$$
\mathbf{u} \sim \boldsymbol{\Gamma} \cdot \mathbf{x}+\frac{m}{2 \pi} \frac{\hat{\mathbf{x}}}{|\mathbf{x}|}+O\left(1 /|\mathbf{x}|^{2}\right), \text { for }|\mathbf{x}| \rightarrow \infty,
$$

where

$$
\Gamma=\frac{1}{2}\left(\begin{array}{cc}
\alpha_{0} & \beta_{0}-\omega_{0} \\
\beta_{0}+\omega_{0} & -\alpha_{0}
\end{array}\right)
$$

Here $\omega_{0}$ is the vorticity of the external flow, while $\alpha_{0}$ and $\beta_{0}$ characterize its strain.

It should also be noted at this stage that the problem stated above can be recast in terms of nondimensional quantities if we rescale velocities by $\sigma / \mu$, pressure by $\sigma / R$, and the length and time scales by $R$ and $R \mu / \sigma$, where $\pi R^{2}$ is the bubble initial area. In these units, the dimensionless parameters characterizing the problem are

$$
\Gamma^{\prime}=\frac{\mu R}{\sigma} \Gamma \text { and } m^{\prime}=\frac{\mu}{\sigma R} m
$$


We prefer however to use dimensionful quantities throughout the rest of the paper, except where noted otherwise. This is because a different nondimensionalization is appropriate when $\sigma=0$.

As is well known (Lamb 1945), the problem of two-dimensional Stokes flow can be conveniently formulated in terms of a stream function $\psi(x, y)$, defined as

$$
u_{1}=\frac{\partial \psi}{\partial y}, u_{2}=-\frac{\partial \psi}{\partial x}
$$

so that $\psi$ relates to the fluid vorticity $\omega$ through

$$
\nabla^{2} \psi=-\omega
$$

and obeys the biharmonic equation

$$
\nabla^{4} \psi=0 .
$$

Alternatively, one can formulate this problem in terms of a stress function $\phi(x, y)$ (Muskhelishvili 1963), defined via

$$
\nabla^{2} \phi=\frac{p}{\mu},
$$

so that $\phi$ also obeys the biharmonic equation. Here the time dependence of $\psi$ and $\phi$ has been omitted for notational convenience.

Next we introduce the quantity $W(z, \bar{z})=\phi(x, y)+i \psi(x, y)$, where $z=x+i y$ and the bar denotes complex conjugation. Then according to the Goursat representation for biharmonic functions (Carrier, Krook, and Pearson 1966), W( $z, \bar{z})$ can be written as

$$
W(z, \bar{z})=\bar{z} f(z)+g(z),
$$

where $f(z)$ and $g(z)$ are analytic functions in the fluid region. All the physically relevant quantities can now be expressed in terms of the functions $f(z)$ and $g(z)$. After a little algebra, one can easily establish the following identities:

$$
\begin{aligned}
\frac{p}{\mu}-i \omega & =4 f^{\prime}(z) \\
u_{1}+i u_{2} & =-f(z)+z \bar{f}^{\prime}(\bar{z})+\bar{g}^{\prime}(\bar{z}), \\
e_{11}+i e_{12} & =z \bar{f}^{\prime \prime}(\bar{z})+\bar{g}^{\prime \prime}(\bar{z}) .
\end{aligned}
$$

where prime indicates derivative and $\bar{f}$ denotes the conjugate function: $\bar{f}(z)=\overline{f(\bar{z})}$ (and similarly for $\bar{g}$ ). The functions $f(z)$ and $g^{\prime}(z)$ must also satisfy appropriate boundary conditions, as described below.

First consider the boundary conditions at infinity. From (14) it follows that

$$
f(z) \sim \frac{1}{4}\left[\frac{p_{\infty}(t)}{\mu}-i \omega_{0}\right] z+C(t)+O(1 / z), \text { as }|z| \rightarrow \infty,
$$


where the functions $p_{\infty}(t)$ [the pressure at $\infty$ ] and $C(t)$ are to be determined later. Now using (6), (15), and (17), one finds

$$
g^{\prime}(z) \sim \frac{1}{2}\left(\alpha_{0}-i \beta_{0}\right) z+\bar{C}(t)+\frac{m}{2 \pi z}+O\left(1 / z^{2}\right), \text { as }|z| \rightarrow \infty .
$$

Note that according to (14) and (15) the choice of $C(t)$ does not affect the velocity and pressure fields - a specific choice however will be made below for convenience.

If we now define

$$
N \equiv n_{1}+i n_{2}=i\left(x_{s}+i y_{s}\right)=i z_{s}=i e^{i \theta},
$$

where $s$ is the arclength traversed in the clockwise direction and $\theta$ is the angle between the tangent and the real positive $x$ axis, then the two stress conditions given in (3) can be written as one complex equation:

$$
-p N+2 \mu\left(e_{11}+i e_{12}\right) \bar{N}=\sigma \kappa N .
$$

Using (14), (16), (19), and the fact that $\kappa=-\theta_{s}$, we find after a straightforward calculation that $(20)$ is equivalent to

$$
z_{s} \frac{\partial H(z, \bar{z})}{\partial z}+\bar{z}_{s} \frac{\partial H(z, \bar{z})}{\partial \bar{z}}=-i \frac{\tau}{2} z_{s s}
$$

where $\tau=\sigma / \mu$ and

$$
H(z, \bar{z})=f(z)+z \bar{f}^{\prime}(\bar{z})+\bar{g}^{\prime}(\bar{z}) .
$$

Equation (21) can then be integrated once, yielding the following condition on the bubble surface:

$$
f(z)+z \bar{f}^{\prime}(\bar{z})+\bar{g}^{\prime}(\bar{z})=-i \frac{\tau}{2} z_{s}
$$

Here, without loss of generality, the constant of integration has been set to zero. [This corresponds to a specific choice of $C(t)$.] From (15) and (23), it also follows that on the bubble surface:

$$
u_{1}+i u_{2}=-i \frac{\tau}{2} z_{s}-2 f(z)
$$

Next, we consider the conformal mapping $z(\zeta, t)$ that maps the interior of the unit circle in the $\zeta$ plane to the fluid region (i.e., the exterior of the bubble) in the $z$ plane, such that the $\zeta=0$ corresponds to the point $z=\infty$. We thus write

$$
z(\zeta, t)=\frac{a(t)}{\zeta}+h(\zeta, t)
$$

where $a(t)$ can be chosen real and negative in view of the additional freedom of the Riemann mapping theorem. Here $h(\zeta, t)$ is assumed analytic in $|\zeta| \leq 1$ and such that $z_{\zeta} \neq 0$ in $|\zeta| \leq 1$ at least for some period of time. 
Now recall that the kinematic condition (5) can be written in complex notation as

$$
\operatorname{Im}\left\{\left[Z_{t}-\left(u_{1}+i u_{2}\right)\right] \bar{Z}_{\nu}\right\}=0
$$

where $Z(\nu, t) \equiv z\left(e^{i \nu}, t\right)$. Note also that

$$
z_{s}=\frac{i \zeta z_{\zeta}}{\left|z_{\zeta}\right|}, \text { on }|\zeta|=1
$$

Inserting (24) and (27) into (26) and using the fact that $Z_{\nu}=i \zeta z_{\zeta}$ on $\zeta=e^{i \nu}$, we obtain the following condition on $|\dot{\zeta}|=1$ :

$$
\operatorname{Re}\left[\frac{z_{t}+2 F(\zeta, t)}{\zeta z_{\zeta}}\right]=\frac{\tau}{2\left|z_{\zeta}\right|}
$$

where $\tau=\sigma / \mu$ and

$$
F(\zeta, t)=f(z(\zeta, t), t)
$$

For later use, we note that that in view of (25) the asymptotic condition (17) implies that

$$
F(\zeta, t) \sim \frac{a\left(p_{\infty} / \mu-i \omega_{0}\right)}{4 \zeta}+C(t)+O(\zeta), \text { as }|z| \rightarrow 0 .
$$

We then notice that the quantity within square brackets in (28) is an analytic function of $\zeta$ for $|\zeta| \leq 1$. [Note that the simple poles at $\zeta=0$ in both the numerator and denominator cancel out.] From Poisson formula (Carrier, Krook, and Pearson 1966), it follows that for $|\zeta|<1$ :

$$
z_{t}+2 F(\zeta, t)=\zeta[I(\zeta, t)+i K] z_{\zeta}
$$

where $K$ is a real constant and

$$
I(\zeta, t)=\frac{\tau}{4 \pi i} \oint_{\left|\zeta^{\prime}\right|=1} \frac{d \zeta^{\prime}}{\zeta^{\prime}}\left[\frac{\zeta^{\prime}+\zeta}{\zeta^{\prime}-\zeta}\right] \frac{1}{\left|z_{\zeta}\left(\zeta^{\prime}, t\right)\right|}
$$

By examining the behavior of $(31)$ as $\zeta \rightarrow 0$, we readily obtain

$$
\begin{aligned}
K & =\frac{1}{2} \omega_{0}, \\
p_{\infty}(t) & =-2 \mu\left[I(0, t)+\frac{\dot{a}}{a}\right],
\end{aligned}
$$

where the dot denotes time derivative. Equation (31) can thus be thought of as an evolution equation for the mapping function $z(\zeta, t)$. Indeed, in the next section we shall use this equation to derive several general properties concerning the motion of the singularities of $z(\zeta, t)$ For practical purposes, however, it is more convenient to view (31) as determining $F(\zeta, t)$ [and hence the pressure and vorticity fields] in terms of the conformal mapping. 
Now if we define

$$
G(\zeta, t)=g^{\prime}(z(\zeta, t), t)
$$

then the function $G(\zeta, t)$ can also be expressed in terms of $z(\zeta, t)$ as follows. Inserting (27) into (23), taking the complex conjugate, and using the fact that $\bar{\zeta}=1 / \zeta$ on $|\zeta|=1$, we then find

$$
G(\zeta, t)=-\bar{F}\left(\zeta^{-1}, t\right)-\bar{z}\left(\zeta^{-1}, t\right) \frac{F_{\zeta}(\zeta, t)}{z_{\zeta}(\zeta, t)}+\frac{\tau}{2} \frac{\bar{z}_{\zeta}^{1 / 2}\left(\zeta^{-1}, t\right)}{\zeta z_{\zeta}^{1 / 2}(\zeta, t)}
$$

which is originally valid on $|\zeta|=1$ and therefore elsewhere by analytic continuation. Using (31) to eliminate $F(\zeta, t)$ from the equation above, we obtain after some simplification

$$
\begin{aligned}
G(\zeta, t) & =\frac{1}{2} \bar{z}\left(\zeta^{-1}, t\right)\left\{\frac{z_{\zeta t}(\zeta, t)}{z_{\zeta}(\zeta, t)}-\zeta I_{\zeta}(\zeta, t)-\left[1+\frac{\zeta z_{\zeta \zeta}(\zeta, t)}{z_{\zeta}(\zeta, t)}\right][I(\zeta, t)+i K]\right\} \\
& +\frac{\bar{z}_{\zeta}\left(\zeta^{-1}, t\right)}{2 \zeta}[I(\zeta, t)+i K]+\frac{1}{2} \bar{z}_{t}\left(\zeta^{-1}, t\right) .
\end{aligned}
$$

[In deriving this, we have made use of the relation

$$
I(\zeta, t)+\bar{I}\left(\zeta^{-1}, t\right)=\frac{\tau}{z_{\zeta}^{1 / 2}(\zeta, t) \bar{z}_{\zeta}^{1 / 2}\left(\zeta^{-1}, t\right)},
$$

which follows from the fact that $\operatorname{Re} I(\zeta, t)=\tau / 2\left|z_{\zeta}(\zeta, t)\right|$ on $|\zeta|=1$.]

We point out at this stage that (37) not only gives $G(\zeta, t)$ in terms of $z(\zeta, t)$ but it also determines the evolution of the mapping function itself. In fact, the requirement that the right hand side of (37) is analytic in $|\zeta|<1$, except for a known pole at $\zeta=0$, can be used to determine the time evolution of the parameters in our solutions, as we will see in $\S 4$. Before doing that, however, we would like to describe in the next section the general mathematical properties that underlie the existence of a rather broad class of exact solutions.

\section{General Properties of Singularities}

In this section we shall derive several global properties of the mapping function $z(\zeta, t)$ for arbitrary initial data $z(\zeta, 0)$ analytic in $|\zeta| \leq 1$ (except for a simple pole at $\zeta=0$ ) and with $z_{\zeta}(\zeta, 0) \neq 0$ in $|\zeta| \leq 1$ as well. In particular, we shall be interested in the behavior of the singularities of $z(\zeta, t)$ in $|\zeta|>1$. We begin by analytically continuing (31) into the exterior of the $\zeta$ unit circle. Through a standard procedure of contour deformation, one finds that for $|\zeta|>1$ :

$$
z_{t}+2 F(\zeta, t)=\zeta[I(\zeta, t)+i K] z_{\zeta}+\tau \frac{\zeta z_{\zeta}^{1 / 2}(\zeta, t)}{\bar{z}_{\zeta}^{1 / 2}\left(\zeta^{-1}, t\right)} .
$$

Now on taking the complex conjugate of (36) on the unit $\zeta$ circle, it follows that

$$
\bar{G}\left(\zeta^{-1}, t\right)=-F(\zeta, t)-z(\zeta, t) \frac{\bar{F}_{\zeta}\left(\zeta^{-1}, t\right)}{\bar{z}_{\zeta}\left(\zeta^{-1}, t\right)}+\frac{\tau}{2} \frac{\zeta z_{\zeta}^{1 / 2}(\zeta, t)}{\bar{z}_{\zeta}^{1 / 2}\left(\zeta^{-1}, t\right)}
$$


on $|\zeta|=1$ and elsewhere by analytic continuation. In view of (40), it is clear that (39) can be written as

$$
z_{t}=q_{1} z_{\zeta}+q_{3} z+q_{2}
$$

where

$$
\begin{aligned}
q_{1}(\zeta, t) & =\zeta[I(\zeta, t)+i K] \\
q_{3}(\zeta, t) & =2 \frac{\bar{F}_{\zeta}\left(\zeta^{-1}, t\right)}{\bar{z}_{\zeta}\left(\zeta^{-1}, t\right)} \\
q_{2}(\zeta, t) & =2 \bar{G}\left(\zeta^{-1}, t\right) .
\end{aligned}
$$

Note from the definitions (32) and (42)-(44) that as long as a solution $z(\zeta, t)$ exists for which $z_{\zeta}(\zeta, t)$ is nonzero and analytic in $|\zeta| \leq 1$ each of $\zeta^{-1} q_{1}, q_{2}$ and $q_{3}$ will remain analytic in $|\zeta| \geq 1$. This implies that the corresponding Laurent series on $|\zeta|=1$ will contain only nonpositive powers of $\zeta$. For instance, one has that on the unit circle

$$
q_{2}\left(e^{i \nu}, t\right)=\sum_{n=-\infty}^{0} q_{2_{n}}(t) e^{-i n \nu} .
$$

Similar Fourier series representations exist for $q_{3}\left(e^{i \nu}, t\right)$ and $e^{-i \nu} q_{1}\left(e^{i \nu}, t\right)$.

In order to further elucidate the properties of the evolution equation (41) in $|\zeta| \geq 1$, it is convenient to introduce the following projection operator $\mathcal{P}_{N}$ acting on the class of functions $v(v)$ with a convergent Fourier representation

$$
v(\nu)=\sum_{n=-\infty}^{\infty} v_{n} e^{i n \nu},
$$

where $\mathcal{P}_{N}$ for $N \geq 0$ is defined by

$$
\left[\mathcal{P}_{N} v\right](\nu)=\sum_{n=N+1}^{\infty} v_{n} e^{i n \nu}
$$

On applying the operator $\mathcal{P}_{N}$ to $(41)$, it follows that the projection $H(\nu, t)=\mathcal{P}_{N} z\left(e^{i \nu}, t\right)$ satisfies the following evolution

$$
H_{t}=\mathcal{P}_{N}\left[-i e^{-i \nu} q_{1}\left(e^{i \nu}, t\right) H_{\nu}\right]+\mathcal{P}_{N}\left[q_{3}\left(e^{i \nu}, t\right) H\right]
$$

It follows from (48) that if $H(\nu, 0)=0$, then $H(\nu, t)=0$ for all times. This fact implies in turn that if $z(\zeta, 0)$ has no singularities in $|\zeta| \geq 1$, except for a "pole" of order $N$ at infinity, i.e., $z(\zeta, 0) \sim$ Constant $\cdot \zeta^{N}$ as $\zeta \rightarrow \infty$, then $z(\zeta, t)$ will have the same property for $t>0$. Since $z(\zeta, t)$ is a conformal map with only one possible singularity in $|\zeta|<1$ at $\zeta=0$, it follows that if we start with an initial condition of the form

$$
z(\zeta, 0)=\frac{a(0)}{\zeta}+h(\zeta, 0),
$$


with $h(\zeta, 0)$ a $N$-th order polynomial, then for later time we will have

$$
z(\zeta, t)=\frac{a(t)}{\zeta}+h(\zeta, t)
$$

where $h(\zeta, t)$ will remain a polynomial of the same order. Thus analyticity properties of $\zeta^{-1} q_{1}$, $q_{2}$ and $q_{3}$ in the extended complex plane $|\zeta| \geq 1$ guarantee the existence of polynomial-type exact solutions.

Now, consider more general initial conditions of the form

$$
z(\zeta, 0)=\sum_{j=1}^{n} E_{j}(\zeta, 0)\left(\zeta-\zeta_{j}(0)\right)^{-\gamma_{j}}+M(\zeta, 0)
$$

with $\gamma_{j}$ either $>0$, or $<0$ but not an integer. Then, it is clear that for later times, we will have

$$
z(\zeta, t)=\sum_{j=1}^{n} E_{j}(\zeta, t)\left(\zeta-\zeta_{j}(t)\right)^{-\gamma_{j}}+M(\zeta, t),
$$

provided $M(\zeta, t)$ and $E_{j}(\zeta, t)$ are required to satisfy

$$
\begin{aligned}
& M_{t}=q_{1} M_{\zeta}+q_{3} M+q_{2} \\
& E_{j_{t}}=q_{1} E_{j_{\zeta}}+q_{3} E_{j}+\frac{\gamma_{j} E_{j}}{\zeta-\zeta_{j}}\left[q_{1}(\zeta, t)-q_{1}\left(\zeta_{j}(t), t\right)\right],
\end{aligned}
$$

while the location of the singularities $\zeta_{j}(t)$ is determined from

$$
\dot{\zeta}_{j}=-q_{1}\left(\zeta_{j}(t), t\right)
$$

Using the projection operator $\mathcal{P}_{\mathcal{N}}$ on (53), it can be shown with an argument similar to that following (48) that if $M(\zeta, t)$ is initially analytic in $|\zeta| \geq 1$ with a $N$-th order "pole" at $\infty$, then it will remain so for later times. Similar property can also be shown to hold for each $E_{j}(\zeta, t)$. The analyticity properties of $E_{j}(\zeta, t)$ and $M(\zeta, t)$ thus imply that the form of an initial singularity of $z(\zeta, t)$ in $|\zeta| \geq 1$ (including that at $\infty$ ) is preserved in time.

Moreover, from Plemelj formula applied to $(32)$, it follows that $\operatorname{Re} I(\zeta, t)=-\frac{\tau}{2\left|z_{\zeta}\right|}$ in the limit $|\zeta| \rightarrow 1^{+}$. And since $R e I(\zeta, t)$ defines a harmonic function everywhere in $|\zeta|>1$, the maximum principle for harmonic functions implies that $R e I(\zeta, t)<0$ in $|\zeta|>1$. Using this fact and (42) into (55) it follows that

$$
\operatorname{Re}\left[\frac{\dot{\zeta}_{j}}{\zeta_{j}}\right]=-\operatorname{Re} I\left(\zeta_{j}, t\right)>0
$$

This corresponds to the statement that the singularities $\zeta_{j}$ move outwards away from the unit circle. This property in fact transcends the restriction implicit in the decomposition $(52)$; it actually holds for all singularities. To see this, note that since $q_{1}, q_{2}$ and $q_{3}$ are 
known to be analytic a priori it follows from the general theory of first order linear partial differential equations with analytic coefficients that any singularity of $z(\zeta, t)$ must propagate according to (55). The fact that singularities (if present) move away from the unit circle implies that for a bubble with a smooth initial boundary, no finite-angled corners can form; the only possibility that remains open is for a zero of $z_{\zeta}$ to impinge on $|\zeta|=1$ in finite time causing a zero-angled cusp on the interface. The solutions discussed in subsequent sections suggest that this is not possible when surface tension is present. However, we could not completely rule out this possibility for more general initial conditions.

Now suppose that all the $\gamma_{j}$ 's in (51) are integers, so that $z(\zeta, 0)$ has only a finite collection of poles in $|\zeta|>1$ in addition to the simple pole at $\zeta=0$, i.e., the conformal mapping is initially a rational function. Then it is clear from the discussion above that as long as the solution exists, $z(\zeta, t)$ will remain a rational function analytic in $|\zeta|<1$. In other words, the problem admits exact solutions where $h(\zeta, t)$ in $(50)$ is a rational function. [We remark parenthetically, however, that closed form solutions for arbitrary nonintegral $\gamma_{j}$ are not possible, because $z(\zeta, t)$ is not analytic at $\zeta=1 / \zeta_{j}(t)$ inside the unit circle on secondary Riemann sheets.]

The theoretical discussion in this section thus shows the close connection between the existence of exact solutions and the analyticity of $q_{1}, q_{2}$ and $q_{3}$ outside the unit circle. It is interesting to note that the equations for the interfacial displacement in a Hele-Shaw cell can also be cast in a form similar to (40) when surface tension is neglected (Tanveer 1993). In this case, the equations also admit exact solutions in terms of polynomials or rational functions (see, e.g., Howison 1992 for a review). The difference, however, is that in the Hele-Shaw problem, when a less viscous fluid is injected into a more viscous fluid, all initial singularities in $|\zeta|>1$ (except that at infinity) travel towards $|\zeta|=1$ (Tanveer 1993). On the other hand, when the more viscous fluid displaces the less viscous one, all singularities move outward. In our case, the outward motion of singularities in the $\zeta$ plane (away from the physical interface) is not affected by suction $(m<0)$ or injection of bubble fluid $(m>0)$ and is found to be the same for a drop (Tanveer and Vasconcelos 1994b) as for a bubble. In this sense, the dynamics here is very different from that of a Hele-Shaw cell.

\section{Polynomial Exact Solutions: General Results}

In this section we present a general class of exact solutions for the case in which the function $h(\zeta, 0)$ is a polynomial of degree $N$. Such initial conditions form a dense set on the class of smooth initial shapes, so that any given smooth initial condition can be approximated by such $h(\zeta, 0)$ to any desired accuracy. The arguments of the previous section guarantee that $h(\zeta, t)$ will remain a polynomial of the same order $N$. Because of the outward motion 
of complex singularities for more general initial conditions, it may be expected that the polynomial class of solutions $h(\zeta, t)$ will be dense in the class of all analytic solutions. Hence if we start with a polynomial approximation $h(\zeta, 0)$ to the true initial shape, the corresponding polynomial $h(\zeta, t)$ is expected to remain a good approximation to the actual interface for later times. Thus, the solutions presented below are expected to describe the evolution for essentially any smooth initial bubble shape.

Accordingly, we seek solutions of the form:

$$
z(\zeta, t)=\frac{a(t)}{\zeta}+\sum_{j=1}^{N} b_{j}(t) \zeta^{j}
$$

where the $b_{j}$ 's are complex coefficients [and recall $a(t)<0$ ]. The problem now consists in finding a set of evolution equations for the coefficients $a(t)$ and $b_{j}(t)$. To this end, we first multiply both sides of $(37)$ by $2 z_{\zeta}(\zeta, t)$ to obtain:

$$
\begin{aligned}
2 z_{\zeta}(\zeta, t) G(\zeta, t) & =z_{\zeta}(\zeta, t)\left\{\bar{z}_{t}\left(\zeta^{-1}, t\right)-\zeta^{-1}[I(\zeta, t)+i K] \bar{z}_{\zeta}\left(\zeta^{-1}, t\right)\right\} \\
& +\bar{z}\left(\zeta^{-1}, t\right)\left\{z_{\zeta t}(\zeta, t)-\zeta I_{\zeta}(\zeta, t) z_{\zeta}(\zeta, t)\right. \\
& \left.-\left[z_{\zeta}(\zeta, t)+\zeta z_{\zeta \zeta}(\zeta, t)\right][I(\zeta, t)+i K]\right\}
\end{aligned}
$$

According to (18) and (25), as $\zeta$ approaches zero the singular behavior of the left hand side of $(58)$ is given by

$$
2 z_{\zeta}(\zeta, t) G(\zeta, t) \sim-\frac{a^{2}\left(\alpha_{0}-i \beta_{0}\right)}{\zeta^{3}}-\frac{2 a \bar{C}(t)}{\zeta^{2}}-\frac{m}{\pi \zeta}+O(\zeta), \text { as } \zeta \rightarrow 0 .
$$

Next consider the Taylor series expansion of $I(\zeta, t)$ :

$$
I(\zeta, t)=I_{0}+\sum_{j=1}^{\infty} \hat{I}_{j}(t) \zeta^{j}
$$

where the coefficients $I_{0}$ and $\hat{I}_{j}$ are given by [see (32)]

$$
\begin{aligned}
& I_{0}=\frac{\tau}{4 \pi} \int_{0}^{2 \pi} \frac{d \nu}{\left|z_{\zeta}\left(e^{i \nu}, t\right)\right|} \\
& \hat{I}_{k}=\frac{\tau}{2 \pi} \int_{0}^{2 \pi} \frac{e^{-i k \nu}}{\left|z_{\zeta}\left(e^{i \nu}, t\right)\right|} d \nu, \quad k \geq 1 .
\end{aligned}
$$

To simplify the notation we define $\hat{I}_{0}=I_{0}+i K$. Now let $R(\zeta, t)$ denote the right hand side of (58). Inserting (57) and (60) into (58), and after performing a tedious but straightforward algebra [made easier with the help of a symbolic-computation software such as Maple], we find that the singular behavior of $R(\zeta, t)$ as $\zeta$ approaches zero is given by

$$
R(\zeta, t)=\sum_{j=1}^{N+2} r_{j}(t) \zeta^{-j}+O(\zeta), \text { as } \zeta \rightarrow 0
$$


with the coefficients $r_{k}$ of the form

$$
r_{k}=-\dot{X}_{k}-(k-1) \sum_{j=0}^{N+2-k} \hat{I}_{j} X_{k+j}, \quad 1 \leq k \leq N+2,
$$

where the quantities $X_{k}$ are given by

$$
\begin{aligned}
X_{N+2} & =a b_{N}^{*} \\
X_{N+1} & =a b_{N-1}^{*} \\
X_{k} & =a b_{k-2}^{*}-\sum_{j=1}^{N+1-k} j b_{j} b_{k+j-1}^{*}, \quad 3 \leq k \leq N \\
X_{2} & =-\sum_{j=1}^{N-1} j b_{j} b_{j+1} \\
X_{1} & =a^{2}-\sum_{j=1}^{N} j\left|b_{j}\right|^{2} .
\end{aligned}
$$

Here star stands for complex conjugation.

Comparing (63) with (59) and matching the terms corresponding to the double pole at $\zeta=0$, one obtains the function $C(t)$ in terms of the coefficients $a(t)$ and $b_{k}(t)$ :

$$
\bar{C}(t)=\frac{1}{2 a}\left[\dot{X}_{2}+\sum_{j=0}^{N} \hat{I}_{j} X_{j+2}\right] .
$$

Similarly, by matching the remaining singular terms we obtain the following system of ordinary differential equations (ODE's) :

$$
\begin{aligned}
& \dot{X}_{1}=\frac{m}{\pi} \\
& \dot{X}_{k}=-(k-1) \sum_{j=0}^{N+2-k} \hat{I}_{j} X_{k+j}+a^{2}\left(\alpha-i \beta_{0}\right) \delta_{k 3}, \quad 3 \leq k \leq N+2 .
\end{aligned}
$$

Note that since the area $A$ enclosed by the curve obtained as the image of the unit circle under the mapping (57) is given by $A=\pi X_{1}$, it follows that (71) simply recovers the condition $\dot{A}=m$. Equations (71) and (72) in general give a system of $2 N+1$ ODE's from which one can compute the $2 N+1$ parameters of the conformal mapping for specified initial data and external flow. In the rest of the paper we will discuss several particular cases of interest.

\section{Bubble in a linear flow}

Throughout this section we assume that the bubble has a constant volume so that we set $m=0$. The simplest scenario is obtained when there is no imposed external flow, in which 
case all initial shapes relax to a circle. Here however we shall consider the more interesting case in which the bubble is placed in a linear flow. Although the general solutions described in $\S 4$ can handle a large class of initial shapes, for simplicity we shall focus here on the case where the bubble possesses an initial circular shape. Below we present exact solutions describing the subsequent bubble evolution (deformation) for two specific flows: (i) pure straining flow and (ii) simple shear flow.

\subsection{Pure straining flow}

Here we imagine that the bubble is placed in a pure straining flow: $\mathbf{u}_{0}=(Q x,-Q y)$, where $Q$ is the rate of shear. In view of (7), we thus set $\alpha_{0}=2 Q$ and $\beta_{0}=\omega_{0}=0$. Under the assumption that the initial shape is a circle of radius $R$, one can easily verify from the general solutions presented above that for later times the interface will be described by a conformal mapping of the form

$$
z(\zeta, t)=\frac{a(t)}{\zeta}+b(t) \zeta
$$

where $b(t)$ is real. From (71) and (72) one finds that the time evolution of the coefficients $a$ and $b$ will be governed by the following ODE

$$
\frac{d}{d t}(a b)=-2 I_{0} a b+Q a^{2},
$$

with $a(0)=R$ and $b(0)=0$, together with the area condition

$$
a^{2}-b^{2}=R^{2}
$$

Here the quantity $I_{0}$ reads [see (61)]

$$
I_{0}=\frac{\tau}{2 \pi} \int_{0}^{\pi} \frac{d \nu}{\left\{a^{2}+b^{2}-2 a b \cos \nu\right\}^{1 / 2}} .
$$

In Fig. 1 we have integrated (74) in nondimensional units for the case $Q^{\prime}=R Q / \tau=0.75$. We thus see that (starting from a circle) the bubbles will evolve through a series of elliptical shapes towards a steady solution, whose parameters $a_{0}$ and $b_{0}$ are given by the solutions to the equation

$$
Q a-2 I_{0} b=0,
$$

subject to the condition (75). This steady solution was first found by Richardson (1968) through a direct steady-state calculation.

\subsection{Simple shear flow}

Now we consider the case of a bubble placed in a simple shear flow: $\mathbf{u}_{0}=(\Gamma y, 0)$, where $\Gamma$ is the shear strength. Accordingly, we set $\alpha_{0}=0$ and $\beta_{0}=-\omega_{0}=\Gamma$. If the initial shape 
is a circle we then have, as before, that the subsequent shapes are given by the conformal mapping (73), but where now $b(t)$ is a complex coefficient. According to (72), the time evolution is given by the following $\mathrm{ODE}$

$$
\frac{d}{d t}(a b)=-\left(2 I_{0}+i \Gamma\right) a b+i \Gamma a^{2}
$$

plus the area condition

$$
a^{2}-|b|^{2}=R^{2}
$$

Here $I_{0}$ reads

$$
I_{0}=\frac{\tau}{4 \pi} \int_{0}^{2 \pi} \frac{d \nu}{\left\{a^{2}+|b|^{2}-2 a b_{R} \cos \nu+2 a b_{I} \sin \nu\right\}^{1 / 2}}
$$

where $b_{R}$ and $b_{I}$ are the real and imaginary parts of $b$, respectively.

Now defining $X=a b_{R}$ and $Y=a b_{I}$, one readily sees that (78) yields the following system of ODE's:

$$
\begin{aligned}
\dot{X} & =-\left(2 I_{0} X+\Gamma Y\right) \\
\dot{Y} & =-\left(2 I_{0} Y+\Gamma X\right)+\Gamma a^{2} .
\end{aligned}
$$

The steady solution in this case is thus clearly given by the solution to the equations

$$
\begin{array}{r}
2 I_{0} b_{R}+\Gamma b_{I}=0 \\
2 I_{0} b_{I}+\Gamma b_{R}-\Gamma a=0
\end{array}
$$

subject to the constraint (79). [This steady solution, in a somewhat different notation, was also first obtained by Richardson (1968).] In Fig. 2 we show the evolution of interface towards the steady solution for the case $\Gamma^{\prime}=R \Gamma / \tau=1$.

\section{Contracting/expanding bubbles in a quiescent flow}

In this section, we suppose that the bubble is placed in an otherwise quiescent flow, i.e., we set $\alpha_{0}=\beta_{0}=\omega_{0}=0$. For simplicity, the expansion or contraction rate $m$ will be taken to be a constant throughout this section. We also assume that the bubble is symmetrical with respect to the $x$ axis so that the coefficients $b_{j}$ 's are all real. In this case, (72) in general gives a system of $N$ ODE's, which together with the area condition (71) determine the $N+1$ coefficients of the conformal mapping. Before discussing the general case, however, we will first consider the simpler case in which the initial shape has either elliptical or $n$-th fold symmetry. 


\section{1. 'Symmetrical' bubbles}

Here we seek solutions of the form

$$
z(\zeta, t)=\frac{a(t)}{\zeta}+b_{N}(t) \zeta^{N}
$$

where $b_{N}(t)$ is real and assumed for definiteness to be negative. Thus, for $N=1$ the bubble is an ellipse, whereas for $N>1$ it posses $(N+1)$-th fold symmetry. We also note that in this case $\hat{I}_{k}$ vanishes identically for $1 \leq k \leq N+1$. Using this fact on (72), it then follows that if the $b_{k}$ 's are initially zero for $k=1, \ldots, N-1$, they will remain so for later times, thus guaranteeing that a mapping of the form (85) does indeed give an exact solution. [We remark parenthetically however that this is not case, for example, when the bubble is placed in an external flow.] The evolution equation in this case is [see (72)]

$$
\frac{d}{d t}\left(a b_{N}\right)=-(N+1) I_{0} a b_{N},
$$

while the area condition (71) after integration reads

$$
A(t)=\pi\left[a^{2}-N b_{N}^{2}\right]=[A(0)+m t],
$$

where $A(0)$ is the initial bubble area. Here $I_{0}$ can be written as

$$
I_{0}=\frac{\tau}{2 \pi} \int_{0}^{\pi} \frac{d \nu}{\left\{a^{2}+N^{2} b_{N}^{2}-2 N a b_{N} \cos \nu\right\}^{1 / 2}} .
$$

as one can easily verify by inserting (85) into (61) and performing a trivial change of variables.

Note also that a linear stability analysis of (86) readily shows that an expanding circle is stable whereas a contracting one is not. To analyze the behavior of the solutions above in more details, it is convenient to study the motion of the critical points of the conformal mapping, i.e., the points at which $z_{\zeta}(\zeta, t)$ vanishes. Denoting such points by $\zeta_{0 k}, k=0, \ldots, N$, we clearly have: $\zeta_{0 k}=\left[\rho e^{i 2 \pi k}\right]^{1 /(N+1)}$, where $\rho=a / N b_{N}$. Using (86) and (87), we find that the quantity $\rho$ evolves according to the following equation

$$
\dot{\rho}=\frac{\rho\left(N \rho^{2}-1\right)}{N \rho^{2}+1}\left[(N+1) I_{0}+\frac{m}{A(t)}\right] .
$$

We also note, for later use, that in terms of $\rho$ and $A(t)$ the coefficients $a$ and $b_{N}$ read

$$
b_{N}^{2}=\frac{a^{2}}{N^{2} \rho^{2}}=\frac{A(t)}{N\left(N \rho^{2}-1\right)} .
$$

In the case of an expanding bubble $(m>0)$, we immediately see from (89) that $\rho$ increases monotonically with time, so that the zeros $\zeta_{0 k}$ move away from the unit circle 
$(|\zeta|=1)$. Hence the bubble has a tendency to become 'smoother' with time and asymptotes an expanding circle as $t \rightarrow \infty$. An example of such a case is presented in Fig. 3 where we show a sequence of interface shapes for $N=1$ (elliptical bubbles) and $m^{\prime}=1$ [see (8)]. It should also be noted that the results discussed in this paragraph are true in general, that is, for any given initial shape [described by a polynomial $h(\zeta, 0)$ ] the bubble will approach an expanding circle as $t \rightarrow \infty$. [Note, in particular, that this is true even in the absence of surface tension.]

Consider now the case of a contracting bubble, i.e., $m<0$. Here, of course, solutions can exist at most up to the time $t_{f}=A(0) /|m|$, at which the bubble-fluid ('air') would be completely removed from the liquid. We will see below that in the absence of surface tension $(\tau=0)$, the solutions will in general breakdown before this time, due to the formation of cusp-singularities on the bubble surface. For $\tau \neq 0$, however, the solutions will always exist all the way up to the final time $t_{f}$.

We first consider the zero surface tension case. Setting $I_{0}=0$ in (89) and integrating the resulting equation, one finds

$$
\rho(t)=\gamma \hat{t}+\frac{1}{N} \sqrt{1+N^{2} \gamma^{2} \hat{t}^{2}}
$$

where $\hat{t}=t_{f}-t$ and

$$
\gamma=-\frac{m}{2 \pi N a(0) b_{N}(0)}>0 .
$$

Here $a(0)$ and $b_{N}(0)$ are the prescribed initial data. Thus, in this case the zeros will hit the unit circle at a time $t_{c}=t_{f}-\pi(N-1) / 2 N \gamma$, since $\rho\left(t_{c}\right)=1$. For $N=1$ (elliptical bubbles), we then see that the zeros hit the unit circle at precisely the time when the bubble area goes to zero. Note that since $a\left(t_{f}\right)=b\left(t_{f}\right) \neq 0$ [see (90)] the final stage of the bubble in this case is a slit of extension $2\left|a\left(t_{f}\right)\right|=2 \sqrt{a(0) b_{1}(0)}$. On the other hand, for $N>1$ the zeros impinge on the unit circle at $t=t_{c}<t_{f}$, leading to the formation of cusps on the interface and hence the breakdown of the solutions before the air is completely removed.

A non-zero surface tension is expected, on general grounds, to prevent the development of actual cusp by providing a 'regularization' mechanism. Indeed, one of the advantages of the solutions above is that they are simple enough to allow a detailed analytical investigation of the regularizing effects of surface tension. For example, recalling that $I_{0}$ diverges if a zero of $z_{\zeta}(\zeta . t)$ lies on the $\zeta$ unit circle $(|\zeta|=1)$, we immediately see from (89) that the zeros must approach $|\zeta|=1$ in the limit that the area vanishes, but they cannot hit the unit circle while the area remains finite. Furthermore, here it is also possible to carry out an asymptotic analysis of the final stages of the bubble evolution, as indicated below. [A similar study has been recently performed by Howison and Richardson (1994) for the case of blob of viscous fluid with suction, where exact solutions with an analogous structure have also been found.] 
We begin our analysis by noting [see the Appendix] that the leading-order asymptotics of $I_{0}$ in the limit $\rho \rightarrow 1^{+}$is given by

$$
I_{0} \approx-\frac{\tau}{2 \pi}\left[\frac{N \rho^{2}-1}{N A(t)}\right]^{1 / 2} \ln (\rho-1)+O(1) .
$$

Inserting this into (89) and writing $A(t)=-m \hat{t}$, one finds that as $\rho \rightarrow 1$

$$
\dot{\rho} \approx-\frac{\rho\left(N \rho^{2}-1\right)}{N \rho^{2}+1}\left\{\frac{\tau(N+1)}{2 \pi}\left[\frac{N \rho^{2}-1}{-N m \hat{t}}\right]^{1 / 2} \ln (\rho-1)+\frac{1}{\hat{t}}\right\} .
$$

The asymptotic behavior of $\rho(t)$ for $\hat{t} \rightarrow 0$ can now be calculated by balancing the most singular terms in (94). Here there are two distinct cases to consider: (i) $N=1$ and (ii) $N>1$.

For $N=1$ (elliptical bubbles), the contribution arising from surface tension effects [i.e., the first term in the right hand side of (94)] is small compared to the other terms, so that effectively the time evolution of the solutions is described by

$$
\dot{\rho} \approx-\frac{\left(\rho^{2}-1\right)}{2} \frac{1}{\hat{t}}, \text { as } \hat{t} \rightarrow 0
$$

which upon integrating yields

$$
\rho \sim 1+\delta \hat{t}, \text { as } \hat{t} \rightarrow 0
$$

for some positive $\delta$. Now according to (90), a linear behavior in $\rho(t)$ as $\rho \rightarrow 1$ implies that $a\left(t_{f}\right)=b\left(t_{f}\right) \neq 0$, so that elliptical bubbles will shrink to a slit even when $\tau \neq 0$. Here the effect of surface tension is simply to reduce the size of such a slit. In other words, the larger the surface tension, the smaller the extension of the final slit.

For $N>1$, as the zeros approach the unit circle, surface tension effects tend to slow down their motion, so that 'narrow structures' (near-cusps) are formed on the bubble surface. An example of this process is given in Fig. 4, where we show a sequence of interface shapes for $N=3$ and $m^{\prime}=-1$, up to the formation of near-cusps. The ensuing 'slow' dynamics of the zeros, as the bubble continues to contract, can be estimated by balancing the two leading-order terms in the right hand side of (94), that is,

$$
\frac{\tau(N+1)}{2 \pi}\left[-\frac{N-1}{N m \hat{t}}\right]^{1 / 2} \ln (\rho-1) \sim-\frac{1}{\hat{t}}
$$

Solving this for $\rho$ yields

$$
\rho \sim 1+e^{-\delta / \sqrt{\hat{t}}}, \text { as } \hat{t} \rightarrow 0
$$

where

$$
\delta=-\frac{2 \pi}{\tau(N+1)}\left[-\frac{N m}{N-1}\right]^{1 / 2}
$$


Note that, as a consistency check, one can now use (98) to verify that the left hand side of (94) is indeed small compared to the leading-order terms on the right hand side, as initially assumed in the argument above.

To obtain a qualitative description of the asymptotic shapes as $\hat{t} \rightarrow 0$, let us denote by $R_{m a x}$ and $R_{m i n}$ the maximum and minimum radial distance of a point on the interface (relative to the bubble center). From (85), one readily finds that $R_{\max }=|a+b|$ and $R_{\min }=|a-b|$. Defining the deformation $D=R_{\min } / R_{\max }$, we then have

$$
D=\frac{N \rho-1}{N \rho+1} \text {. }
$$

Since the deformation $D$ goes to a constant in the limit $\rho \rightarrow 1$, it follows that during the late stages the bubble will shrink to a point through a succession of geometrically similar shapes. [An analogous phenomenon has also been observed by Howison and Richardson (1994) for the case of a blob of viscous fluid with suction.] Here the main effect of surface tension is to determine the time scale for when the 'near-cusps' first appear, that is, the greater the surface tension, the later the near-cusp will develop.

\section{2. 'Asymmetrical' bubbles}

We have seen above that in the case that the initial shape can be described by a conformal mapping of the type given in (57), surface tension effects guarantee that the solutions will always exist up to $t=t_{f}$, by which time the air will have been completely removed from the liquid. The situation changes considerably, however, when the bubble initially has neither elliptical nor $n$-th fold symmetry, that is, when not only $b_{N}(0) \neq 0$ (for $N>1$ ) but also $b_{k}(0) \neq 0$ for some $k<N$. In such cases, the bubble will invariably develop a 'thin neck' whose width will go to zero at a time $t=t_{b}<t_{f}$, after which the solutions cease to make physical sense. We thus refer to this process as bubble 'breakup'. In Fig. 5 we show a sequence of interfaces shapes leading to the breakup of the contracting bubble into three smaller ones. In the case shown in this figure, the coefficients $b_{1}, b_{3}$ and $b_{5}$ were all initially nonzero and $m^{\prime}=-1$. We also found a similar breakup when we take $m$ to be proportional to the bubble perimeter instead of a constant, as appropriate for modeling a dissolving bubble gas.

Since our solutions breakdown at the time when the two sides of the interface 'touch' each other, we are unable to follow the dynamics of the 'newborn' bubbles. We emphasize, however, that no physical quantity blows up as the bubble approaches breakup. Here the breakdown of the solutions is caused simply by the loss of univalence of the conformal mapping for $t>t_{b}$ (Tanveer \& Vasconcelos 1994a). 


\section{Conclusions}

We have presented exact solutions for a bubble with essentially arbitrary initial shape evolving in a two-dimensional Stokes flow in the presence of surface tension. Our solutions, which are given in terms of a polynomial-type mapping function, include bubbles in a linear flow as well as an expanding or contracting bubble in an otherwise quiescent flow. It has been noted that the expanding bubble approaches a growing circle for later times, while a contracting circular bubble is unstable to disturbances and can lead to the formation of nearcusps or cause breakup before all the bubble-fluid is removed. The mathematical structure underlying the existence of a broad class of exact solutions has also been discussed in detail.

As is well known, an inviscid bubble does not breakup under the action of a shear flow. Our results show however that breakup does occur (at least in plane flows) when another driving mechanism, e.g., suction of bubble-fluid, is present. While this general tendency to breakup is likely to be present in 3D axisymmetric flow as well, it not clear at this point that different parts of the interface will actually touch or merely tend to each other. Also, in our solutions the flow inside the bubble has been neglected since the viscosity of the inner fluid has been assumed to be negligible. Nonetheless, this flow is likely to be important near bubble breakup. The effect of a nonzero viscosity ratio thus needs to be examined in the future.

One of the authors (GLV) would like to acknowledge financial support from The Ohio State University Postdoctoral Fellowship. This research was supported in part by DOE contract DE-FG02-92ER14270. 


\section{Appendix}

The asymptotic behavior of the quantity $I_{0}$ given in (88) in the limit $\rho \rightarrow 1$ can be computed in the following way. First we use (90) to rewrite (88) in terms of $\rho$ :

$$
I_{0}=\frac{\tau}{2 \pi}\left[\frac{N \rho^{2}-1}{N A(t)}\right]^{1 / 2} \tilde{I}_{0}(\rho)
$$

where

$$
\tilde{I}_{0}(\rho)=\int_{0}^{\pi} \frac{d \theta}{\left\{1+\rho^{2}-2 \rho \cos (\theta)\right\}^{1 / 2}}
$$

We now separate this last integral into two contributions:

$$
\tilde{I}_{0}(\rho)=\int_{0}^{\epsilon} \frac{d \theta}{\left\{(\rho-1)^{2}+2[1-\cos (\theta)]\right\}^{1 / 2}}+\int_{\epsilon}^{\pi} \frac{d \theta}{\left\{(\rho-1)^{2}+4 \rho \sin ^{2}\left(\frac{\theta}{2}\right)\right\}^{1 / 2}}
$$

where $\rho-1<<\epsilon<1$. Here we have rewritten the integrand in both integrals above in a form suitable for the next step in the calculation. Accordingly, in the first integrals the leading-order asymptotics can be found by approximating $\cos \theta$ by $1-\frac{1}{2} \theta^{2}$ and then performing a change of variable $\theta=(\rho-1) \nu$. Similarly, in the second integral the leadingorder asymptotics is obtained by simply setting $\rho=1$. Within these approximations, one then has

$$
\begin{aligned}
\tilde{I}_{0}(\rho) & \approx \int_{0}^{\epsilon} \frac{d \nu}{\left[(\rho-1)^{2}+\nu^{2}\right]^{1 / 2}}+\int_{\epsilon}^{\pi} \frac{d \nu}{2 \sin \frac{1}{2} \nu} \\
& =\ln \left[\frac{\epsilon}{\rho-1}+\sqrt{1+\frac{\epsilon^{2}}{(\rho-1)^{2}}}\right]-\ln \tan \frac{1}{4} \epsilon
\end{aligned}
$$

In the leading-order the $\epsilon$ dependence drops out from the above, thus giving

$$
\tilde{I}_{0}(\rho) \approx-\ln (\rho-1)+O(1)
$$

Finally, inserting this into Eq. (101) yields formula (93). 


\section{REFERENCES}

Acrivos, A. (1983) The breakup of small drops and bubbles in shear flows. Ann. NY Acad. Sci. 404, 1-11.

Buckmaster, J. D. \& Flaherty, J. E. (1973) The bursting of two-dimensional drops in slow viscous flow. J. Fluid Mech. 60, 625-639.

Carrier, G. F., Krook, M., \& Pearson, C. E. (1966). Functions of a Complex Variable. McGraw-Hill.

Hopper, R. W. (1990) Plane Stokes flow driven by capillarity on a free surface. J. Fluid Mech. 213, 349-375.

Hopper, R. W. (1991) Plane Stokes flow driven by capillarity on a free surface, Part 2: Further developments. J. Fluid Mech. 230, 355-364.

Hopper, R. W. (1992) Stokes flow of a cylinder and a half-space driven by capillarity. $J$. Fluid Mech. 243, 171-181.

Hopper, R. W. (1993) Capillarity-driven plane Stokes flow exterior to a parabola. Q. Jl. Mech. appl. Math. 46, 193-210.

Howison, S \& Richardson, S. (1993) Cusp development in free boundaries, and two-dimensional slow viscous flows. Submitted to Eur. J. appl. Math.

Lamb, H (1945) Hydrodynamics. Dover.

Rallison, J. M. (1984) The deformation of small viscous drops and bubbles in shear flows. Ann. Rev. Fluid Mech. 16, 45-66.

Richardson, S. (1968) Two-dimensional bubbles in slow-viscous flow. J. Fluid Mech. 33, 475-493.

Richardson, S. (1992) Two-dimensional slow viscous flows with time-dependent free boundaries driven by surface tension. Eur. J. appl. Math. 3, 193-207.

Stone, H. A. (1994) Dynamics of drop deformation and breakup in viscous fluids. Ann. Rev. Fluid Mech. 26, 65-102.

Tanveer, S. (1993) Evolution of Hele-Shaw interface for small surface tension. Phil. Trans. R. Soc. Lond. 343, 155-204.

Tanveer, S. \& Vasconcelos, G. L. (1994a) Bubble breakup in two-dimensional Stokes flow. Submitted to Phys. Rev. Lett. 
Tanveer, S. \& Vasconcelos, G. L. (1994b) Unpublished notes.

Taylor, G. I. (1932) The viscosity of a fluid containing small drops of another fluid. Proc. R. Soc. Lond. A 138, 41-48.

Taylor, G. I. (1934) The formation of emulsions in definable fields of flow. Proc. R. Soc. Lond. A 146, 501-523. 


\section{FIGURE CAPTIONS}

Fig. 1. Circular bubble placed in a pure straining flow with $Q^{\prime}=0.75$.

Fig. 2. Circular bubble placed in a simple shear flow with $\Gamma^{\prime}=1$.

Fig. 3. Expanding bubble with $m^{\prime}=1$.

Fig. 4. The evolution of a four-fold symmetric bubble for $m^{\prime}=-1$. Note the formation of 'near-cusps' on the innermost interface.

Fig. 5. The evolution of an 'asymmetric' bubble leading to bubble breakup for $m^{\prime}=-1$. 
Figure I

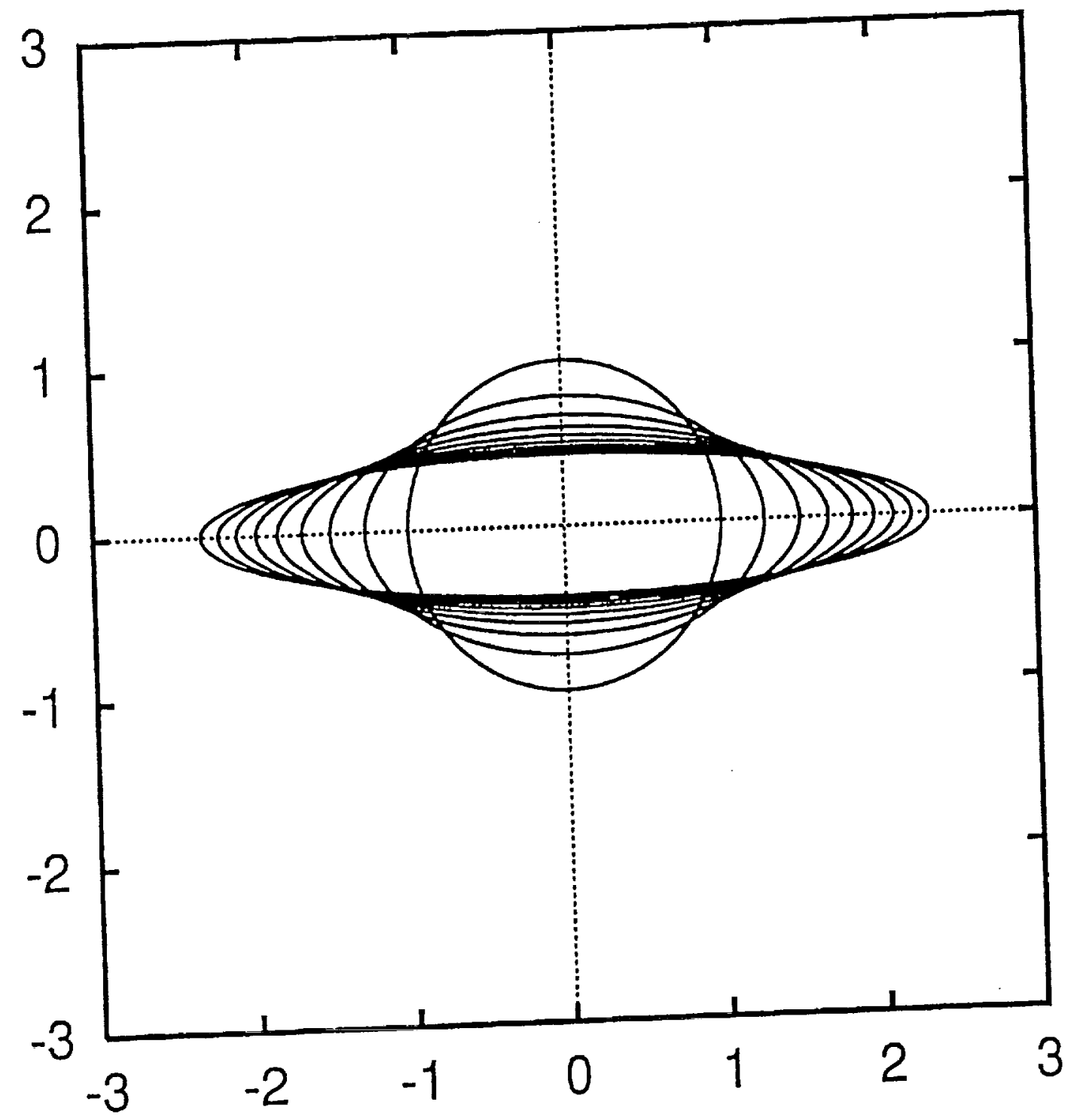


Figure 2

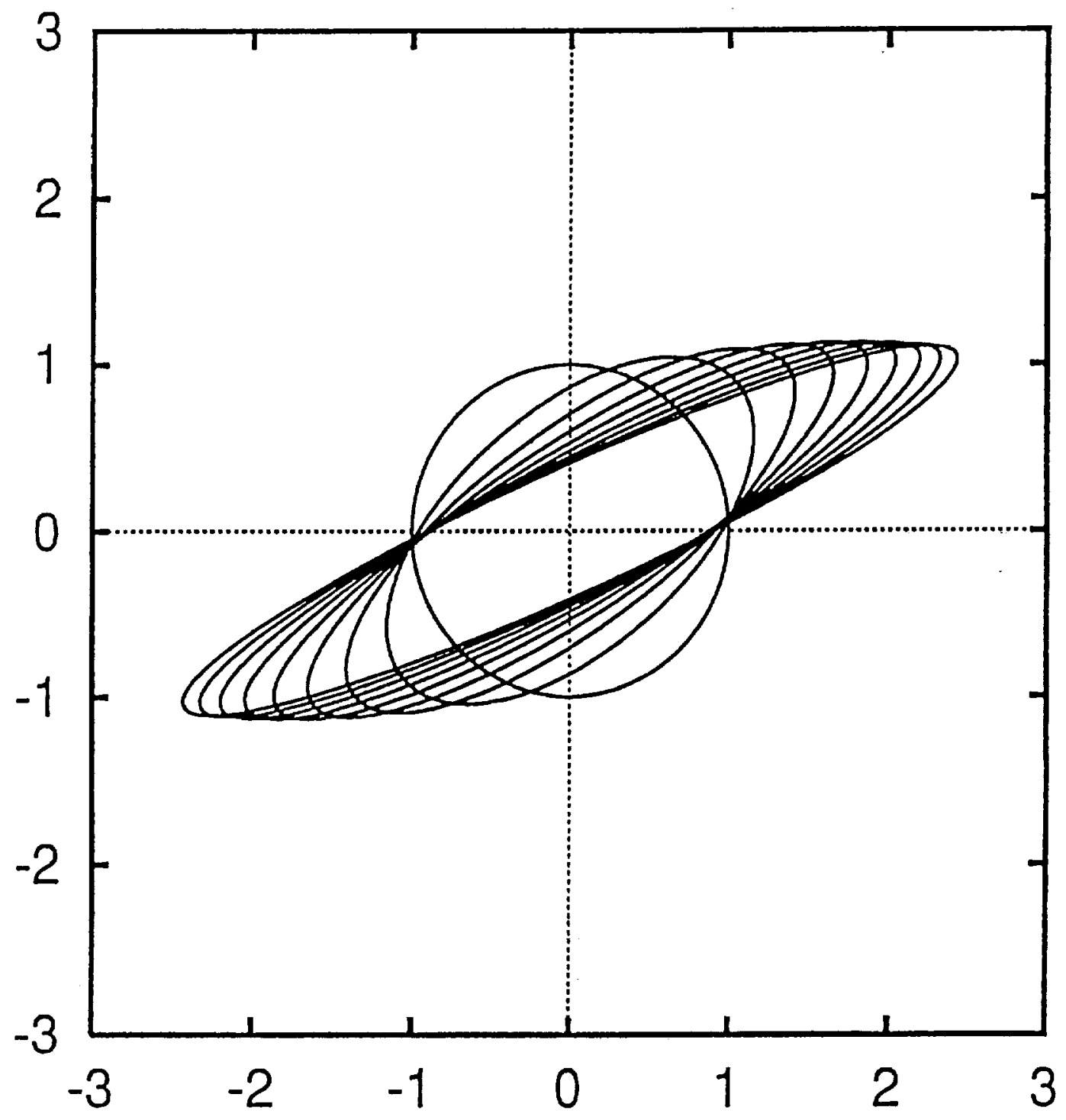


Figure 3

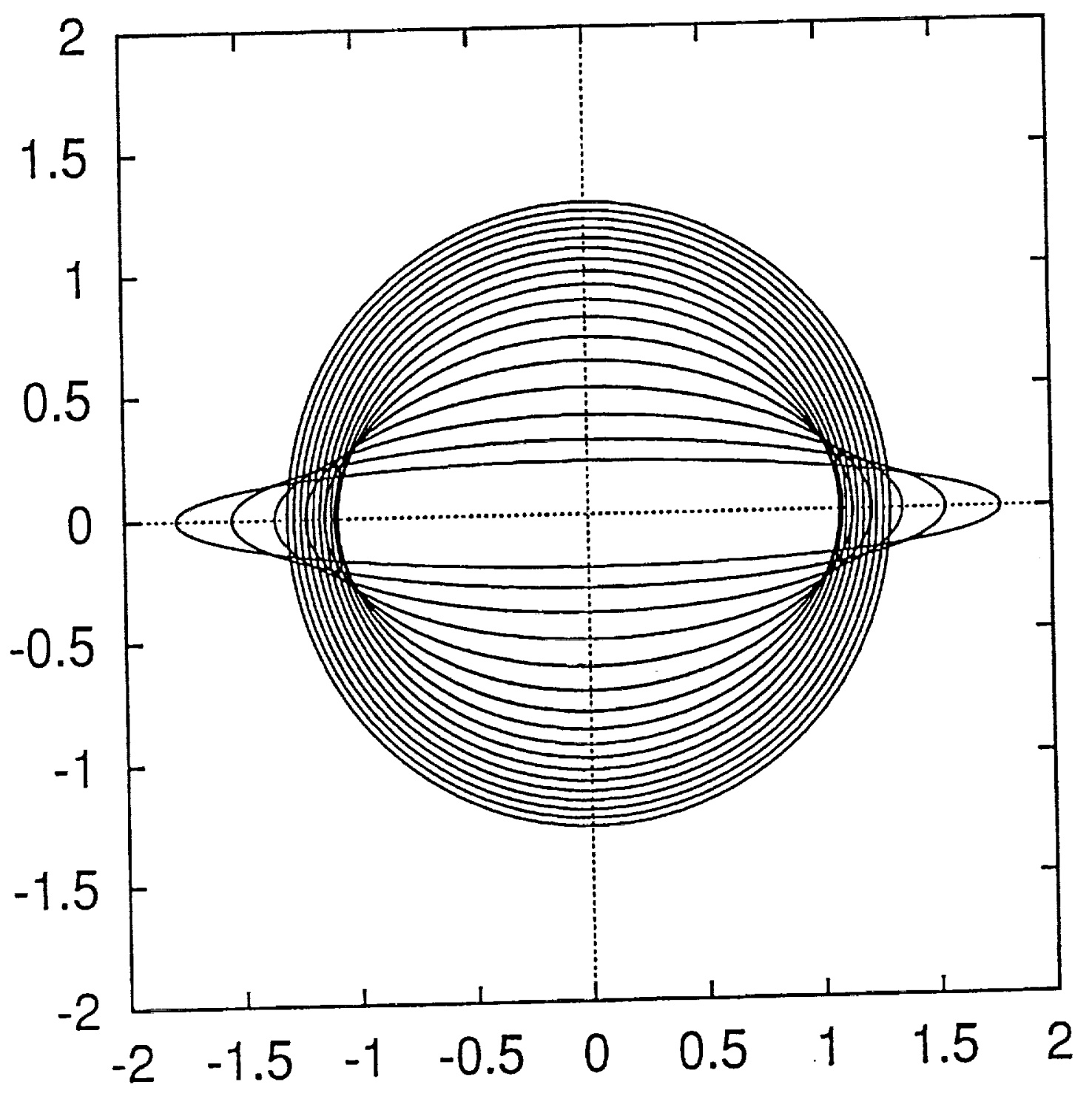


Figure 4

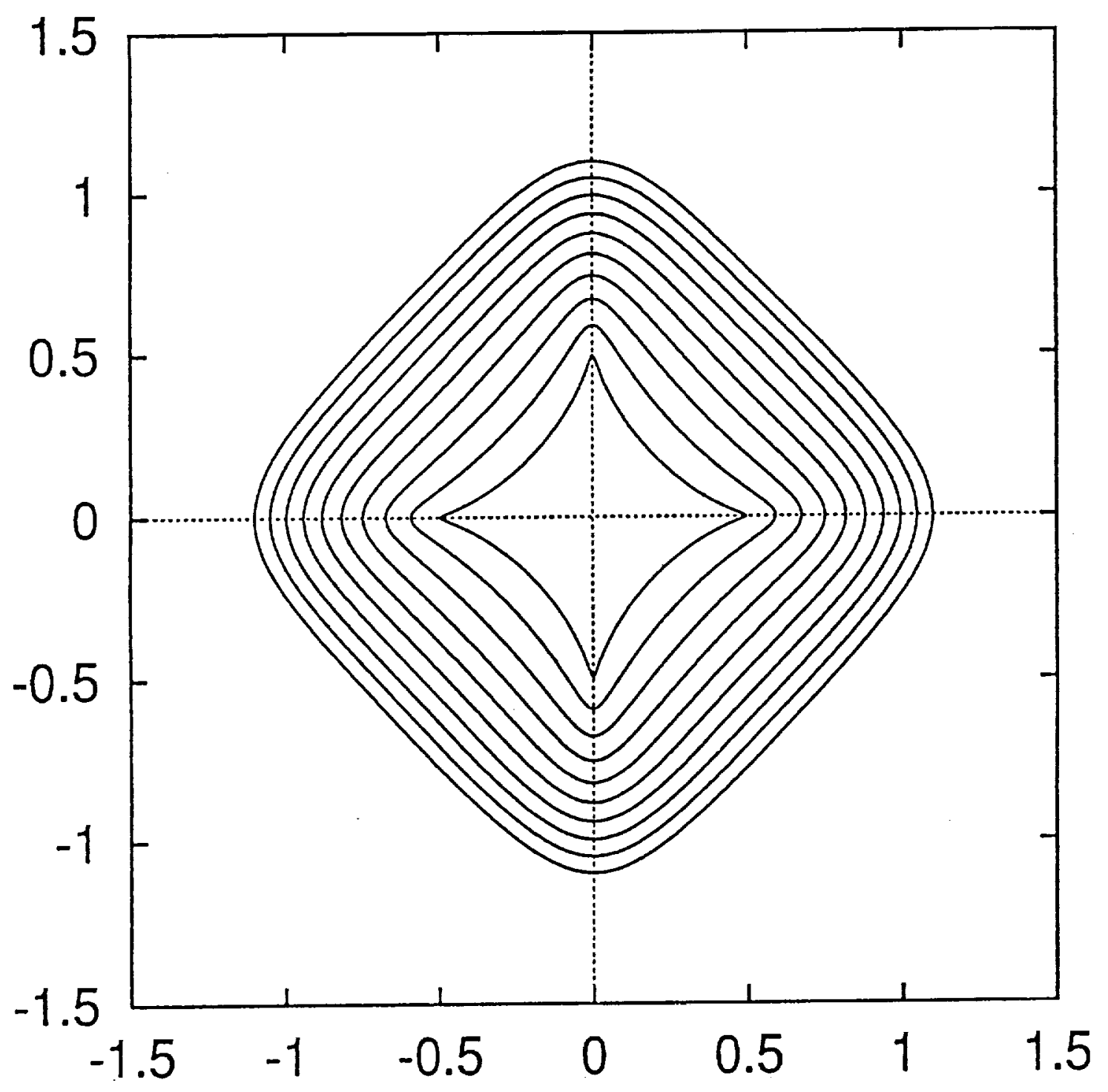


Figure 5

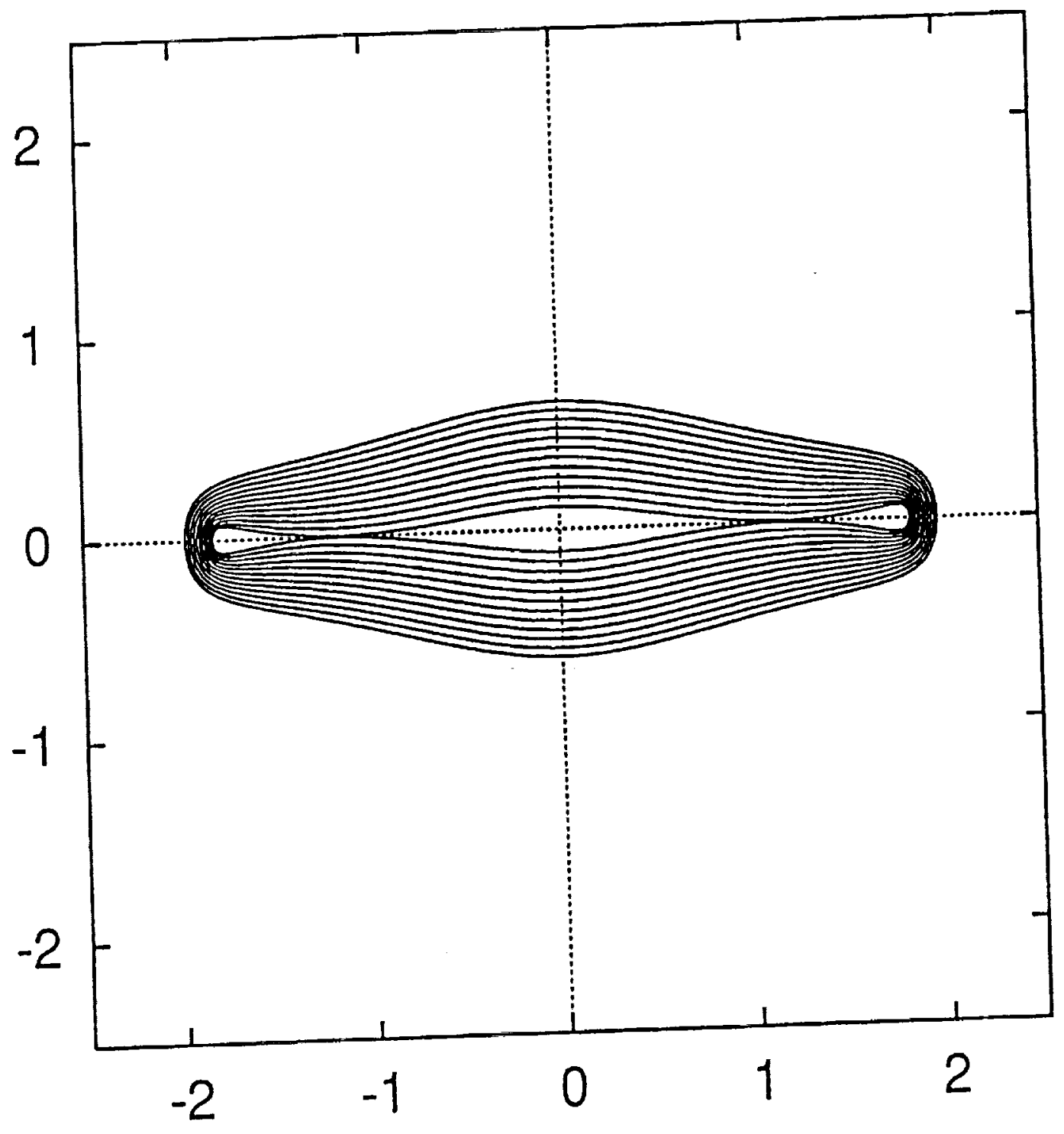




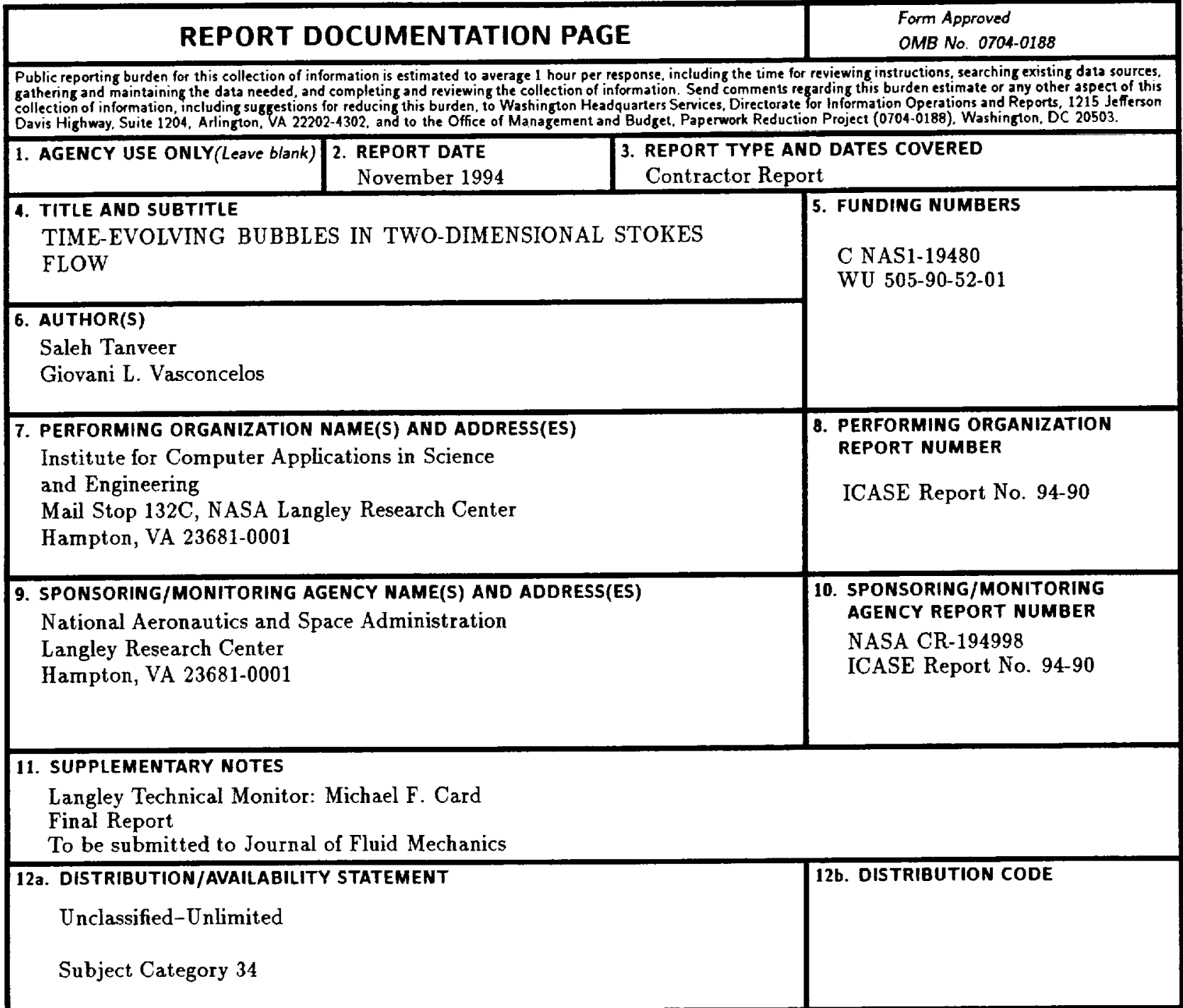

13. ABSTRACT (Maximum 200 words)

A general class of exact solutions is presented for a time evolving bubble in a two-dimensional slow viscous flow in the presence of surface tension. These solutions can describe a bubble in a linear shear flow as well as an expanding or contracting bubble in an otherwise quiescent flow. In the case of expanding bubbles, the solutions have a simple behavior in the sense that for essentially arbitrary initial shapes the bubble will asymptote an expanding circle. Contracting bubbles, on the other hand, can develop narrow structures ('near-cusps') on the interface and may undergo 'break up' before all the bubble-fluid is completely removed. The mathematical structure underlying the existence of these exact solutions is also investigated.

\begin{tabular}{l} 
14. SUBJECT TERMS \\
eXact solutions, Stokes flow \\
\hline $\begin{array}{l}\text { 17. SECURITY CLASSIFICATION } \\
\text { OF REPORT } \\
\text { Unclassified }\end{array}$
\end{tabular}

NSN 7540-01-280-5500
15. NUMBER OF PAGES

30

16. PRICE CODE A03

18. SECURITY CLASSIFICATION 19. SECURITY CLASSIFICATION 20. LIMITATION OF THIS PAGE Unclassified OF ABSTRACT 
